\title{
Reconstructing Geographical Flood Probability and Analyzed Inundation Flood Mapping on Social Media Implementation
}

\author{
Chinnaiyan RAJESHKANNAN ${ }^{1 *}$, Shanmuga Vadivel KOGILAVANI ${ }^{2}$ \\ ${ }^{1}$ Department of Computer Science and Engineering, Suguna College of Engineering, \\ Coimbatore- 641014, Tamil Nadu, India \\ rajeshscholar17@gmail.com (*Corresponding author) \\ ${ }^{2}$ Department of Computer science and Engineering, Kongu Engineering College, \\ Erode- 638060, Tamil Nadu, India \\ kogilavani.sv@gmail.com
}

\begin{abstract}
One of the major issues of the planetary ecosystem is natural disaster prediction, which utilizes the flood prediction approach to a larger extent. Inundation prevention considers the emergency response, post-event damage assessment and flood mitigation. The current reconstructed flood-mapping model is divided into two segments namely the near real span and real span procedure based on the timing information. The inherent conditions of each procedure broadly hamper their application for inundation mapping. This paper considers the South Carolina inundation in Columbia as a previous research work. The proposed research work presents flood reconstruction design by improving near real span normalized water index derived images from the collection of USGS datasets. Real span data is collected from river gauge readings and it is implemented in Social Media (Twitter API). The proposed flood mapping technique is divided into three sections namely the local enhancement section, enhanced water height section and global enhancement section. Here the section synthesis with gauge readings and various normalized water index images (Geoscience information) is presented with a view to reconstructing an advanced macro-scale flood probability condition by means of the Improved Markov Chain Monte Carlo method, which is improved by using the analyzed inundation-related social media. The result matches well with the U.S. Geological Survey flood map and its surveyed high-water marks for flood identification. The results suggest that by enhancing near real span imagery with real span data sources, the proposed flood inundation probability reconstruction model renders a more robust, gradually enhanced flood probability index for emergency responders so that they quickly identify areas in need of urgent attention. The methodology used in this paper could seed a wide range of future flood studies that may lead to a rapid and improved flood situational awareness in a city as well as at a regional level.
\end{abstract}

Keywords: Geographic data science, Data fusion, Reconstructing flood mapping, Probability index, Social Media, MCMC technique.

\section{Introduction}

Flood reconstruction is the most powerful application model on earth, which affects the regular life. Flood mapping approaches are performed with novel algorithms by utilizing data fusion approach. Here the South Carolina immersion in Columbia is considered as data of research work. The flood recreation configuration is obtained by improving a close range for different water list on flood prediction. The water level indication differed based on periods and it is represented by each gauge value of respective water level analysis. The readings are noted and standardized different water levels are used to remake a propelled full-scale flood likelihood condition, which is improved by utilizing internet based lifelike social media sharing. The configurations are coordinated with the U.S.G.S topographical survey and high-water level marked to implement on social media. The USGS geographical data science map provides the flood map with specified gauge numbers (Hung, Wang \& Li, 2018). The snow water equivalent processing system is developed with high- resolution hydrology images; here the spatial resolution product is taken from Glob-Snow and H-SAF portfolios (Takala et al., 2017). Synthetic aperture imaging is applied on the radiometric system; however, the problem of radiometric temperature retrieval system is that it doesn't provide the exact error report (Anterrieu, 2007). Tomographic technique is used for microwave link measurement, which analyses the rainfall rate and predicts the possibility of flood occurrence by overflow. Tsunami scenario and hazard assessment model is presented in (Gailler et al., 2015). Rain gauges are used to identify the rainfall level of a particular area, which indicates the earlier prediction of flood by utilizing various approaches and comparisons (D`Amico, Manzoni \& Solazzi, 2016). Also, ocean model and soil moisture with in-situ properties are taken as data for image reconstruction approach. It measures the effect of polarization on the SMOS (Soil Moisture and Ocean Salinity) image model with different antennas (Landuyt et al., 2018). Reconstruction design of geological approach is performed 
with the dual tree wavelet transformation. Here the non-linear dynamic flow measurement is performed for geological continuity. The Discrete Wavelet Transform coefficient selects the location of different water levels based on the given set of input images of inundation mapping (Evans et al., 2010). The rainfall rate is identified by tracing the error source from the satellite mapping approach, which is evaluated from Tibetan Plateau, China. Heavy rainfall detects the flood possibility by reconstruction model, which detects the error of satellite image on geo spatial model (Zhu et al., 2018). Meteorological operational satellites and RADAR are used for overestimations of rainfall rate (Youngjoo et al., 2014). Root zone is evaluated based on soil moisture data, which is performed on the top-layer of flash flood reconstruction model (Khan et al., 2020). SAR images are used for this flood mapping approach, which is designed with synthetic aperture Radar assessments. Here the pixel-based flood mapping is performed to detect the changes with Support Vector Machine learning (Sarker et al., 2016).

The steering limitations of TSX are discussed and it estimates the different pattern of simulations and its evaluations. The Kepler orbit and WGS84 ellipsoid are the main parameters to estimate the azimuth pattern of the angle. Flood event based on sequence analysis for prediction model using Bayesian estimation is presented in (Xu et al., 2020). Flood inundation test case is surveyed with split-based approach, which performs based on the thresholding of satellite images. Here the flood-mapping algorithm (Chini et al., 2017) identifies the change detection. The machine learning approach is applied for automatic change detection using SAR for flood reconstruction approach. Kittler-Illingworth threshold based criterion is modeled with different classes. Here the Gaussian approach is modeled with filtering process by performing iteration levels (Bazi, Bruzzone \& Melgani, 2005). Flood prediction, monitoring, and rescue system with feature analysis is presented in (Nadeem et al., 2019). Probability of flood mapping is analyzed with the synthetic aperture radar data. Flooded and non-flooded pixels are analyzed with histogram backscattering process (Giustarini et al., 2016). Here the unsupervised approach is used for satellite image classification to detect the flood (Huang, X., Wang, C. \& Li, Z., 2018). The near real-time flood mapping approach is integrated with the post-event process using satellite images
(Huang, X., Wang, C. \& Li, Z., 2018; Li et al., 2017). During the flood event, the tweet points are changed according to the water level, which helps to identify the flooded area by probability assessment with power plants (Gaeta \& Sereno, 2011; Qavi et al. 2017). Here the real-time twitter data helps the people to identify the flooded area (Wang, Huang \& Baetz, 2015) this awareness improves the performance of overall system (Saeed et al., 2018). It utilizes Inception-V3 and embedded CNN model to train the data, which is based on texture features analysis (Shen et al., 2015). Carrier Sense Multiple Access based flood analysis using data fusion technique is presented in (Shah-Mansouri, Pakravan \& Khalaj, 2011; Huang et al., 2018). Textual feature and data fusion of wetness analysis is given in (Wang et al., 2018; Huang et al., 2019). The proposed work has three sectional models, which are designed to perform flood prediction, surveying for earlier flood possibility identification and social media implementations. Water science dataset for flood event prediction is collected (Anon, 2018). The reconstruction model enables the earlier prediction of flood by survey analysis from USGS. A novel approach in this design is to incorporate the three different sections with twitter implementation model. Here the MCMC technique helps to determine the probability of flood, image processing on water index calculation determines the water area of flood region, which can help one notice the most affected flood region; finally, the USGS based flood mapping is implemented on twitter API. This approach was improved by performing with reduced computation time for all the three sectional models of flood reconstructions.

The remainder of this paper is organized as follows. Section 2 describes existing work survey analysis. Section 3 presents the proposed research work with three sectional models and social media implementations. Section 4 discusses the experimental result analysis and computations. Section 5 concludes this paper with the comparison of obtained results and significance of proposed work; finally, the future scope is presented.

\section{Literature Survey}

Hung, Wang \& Li (2018) have proposed the construction of flooding models based on near real-time and real-time gauges. The USGS 
provides the water level data for all the gauge numbers with every 15 -minute interval. Here the gauge sensors are do not record the water height at each instance. Maximum water height readings are translated to elevation on local enhancement model. Here the hardware modules were extracted on local enhancement elevation. This module integrates the digital elevation model and water level points to generate initial flooding probability. The spatially isolated real-time data is used to enhance the captured image. Hence, it provides the continuous view of each gauge records and the twitter post. The drawback of this approach is that it reduces the flooding probability value while running flood map for earlier prediction and it gives only random value.

D' Amico, Manzoni \& Solazzi, (2016) have presented microwave connected system, which was utilized to recreate 2D fields of amassed precipitation over a constrained territory at ground level. Here the tomographic system misuses the connection between precipitation rate and the weakening experienced by the microwave connections. The connections situated in Italy work at K-band and these are overseen by an Internet specialist organization. At last, system performance shows the reconstruction of the flood development model and topology on the exactness of calculations. Sometimes, it may lose the calculation by varying overfitting database.

Landuyt et al. (2018) have discussed about the flood risk with expanding concern on rainy season. The strategies were tested on lesser feature utility based SAR pictures of various flood occasions and lakes over the U.K., and Ireland were assessed on both exactness and power. Because of its high vigor and great execution, tiled thresholding is appropriate for robotized, close constant flood recognition and checking. Dynamic shape models can give higher correctness yet require long calculation times.
Huang et al. (2018) have presented the quick flood mapping of nearby experts and crisis responders to distinguish territories needing prompt consideration. The flood-related tweets utilize quantitative strategies to see how the twitter movement is identified. Outputs illustrate the steady state estimation of the flood circumstances in close constant, which is basic for improving the situational mindfulness in the context of flooding occasion to enable basic leadership.

\section{Proposed Method}

The proposed research work presents the flood reconstruction design by improving a near real span normalized various water index derived from the collection of USGS database. The real span data includes river gauge readings. The flood mapping technique is divided into three sections, which are local enhancement section, enhanced water height module and global enhancement section. Here the synthesis with gauge readings and various normalized water index pictures is presented with a view to reconstructing with an advanced macro-scale flood probability condition, which is improved using analyzed inundation related social media (twitter). The proposed flood reconstruction model (see Figure 1) describes the functions of the local enhancement module, enhanced water height module, and global enhancement module. In local enhancement module, the morphological dilation process by image processing is performed based on the captured global image from USGS map. The enhanced water height module takes the maximum water height regions of each gauge and it performs DEM based filters, which utilizes the twitter API for creating awareness. Prediction is done by flood probability model with the help of 100 year USGS database flood events and tweet API shows the flood web page to identify the flood event in order to raise the awareness of people.

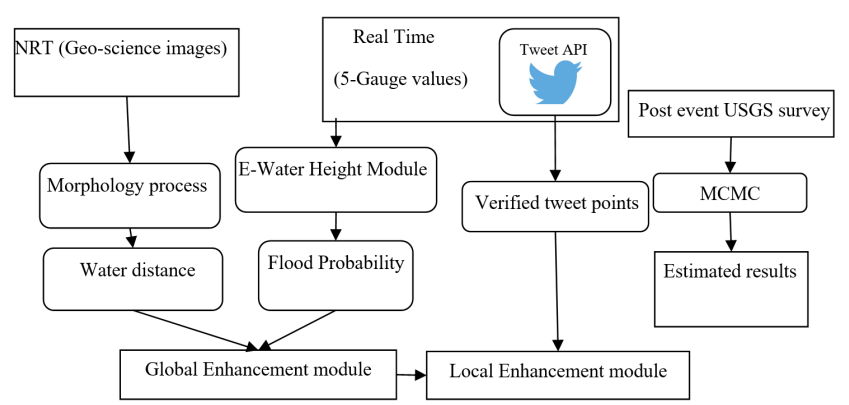

Figure 1. Proposed Flood Reconstruction Model 
Here, the geographical image and gauge data are processed by two segments, which optimize the tweet API to implement the social media. Here the morphological process is applied to find the water index based on the probability of flooding event. Here the gauge readings are measured to identify the exact tweet points and implemented with the twitter API for creating awareness. Major contribution of this research work is to improve the flood probability analysis by three sectional models. Here the improved performance of MCMC and water index determination approaches are implemented and obtain better results by fixed range of average water level determination. All the gauge water levels are aggregated with the filter. Finally, the global enhancement module performs kernel smoothing and a histogram function then it is aggregated on the water area detector model using image processing approach. The probability is found during the flood event. Each tweets point is marked with gauge reading. By using the twitter post, the flood events can be updated based on selectable research area. Here the MCMC technique is applied to survey the flood event based on the USGS data. This method provides the result of USGS survey based on the water distance. The flood frequency peak value determines the flood probability to identify the possibility of flood event. Gauge value with water height value is determined with respect to the discharge value of flood event.

\subsection{Dataset}

The proposed reconstructed model utilizes the USGS database. Here the five gauge readings are taken from that geographical survey and it uses geographical image for analyzing exact location through tweet points. The national water information system is the data portal of USGS real-time data. The USGS collects the flood data and it performs the specific flood science approach for helping local agencies, researchers, and decision makers with regard to flood events in urban areas. After taking the gauge readings, it pointed out by tweets and it has performed flood probability for each tweet point.

The gauges are selected based on the survey requirements; the Washington area based rivers readings are taken for finding the flood event and flood possibilities. Flood possibility is on rivers such as Walla River, Fork Palouse River, Hangman Creek River, Grande Ronde River, and
Snake River. These rivers have a separate gauge number; based on that the flood event is identified earlier. Here the geographical image is taken to analyze for finding the water index for every area, which is utilized to find the exact location.

\subsection{Local Enhancement Section}

In local enhancement section, the probability of flooding layer could be upgraded around the checked flood-related tweets areas, which are the neighborhood points of flood mapping. Concurring to Tobler's law, zones more like a checked tweet point are bound to overflow. The flood index is identified with the flood event and the tweet information is analyzed based on the USGS flood event analysis. The $\mathrm{P}_{\text {flood }}(\mathrm{a}, \mathrm{b})$, forms the function of $f(a, b)$ which is the maximal incentive inside the examiner region balanced by the separation rot impact.

$$
\begin{aligned}
& f(a, b)=\left(\left(P_{\text {flood }} \text { XORe }\right)\right) \\
& f(a, b)=\max \left\{P_{\text {flood }}(a-p, b-q) \in D s ;(p, q) \in D e\right\}
\end{aligned}
$$

Where $\mathrm{D}_{\mathrm{e}}$ signifies the space of selected component e, the last likelihood $\operatorname{RFP}\left(\mathrm{a}, \mathrm{b}_{\mathrm{y}}\right)$ is then determined by presenting a separation-related coefficient ' $\mathrm{m}$ ', which is given by,

$R F_{\text {prob }}(a, b)=\frac{f(a, b)-P_{\text {flood }}(a, b)}{m}+P_{\text {flood }}(a, b)$

Where $\mathrm{m}=(\mathrm{r} /(\mathrm{r}-\mathrm{d}))$ ', from this, ' $\mathrm{r}$ ' represents the sweep of the hunt region and $d$ represents the separation from $(a, b)$ to the tweet point distance. The radius $r$ is set to $1000 \mathrm{~m}$ for long slope. Here the pursuit range is set at approximately $1000 \mathrm{~m}$ for long focus, which performed on the image analysis approach. However, for gauge readings based data, it utilized the overall area based on gauge number coverage. Tweet point in local enhancement module is verified by the flooding probability index; this determination gives the maximum water level point. Tweet points of each gauge enhance the flooding probability.

\subsection{Enhanced Water Height Module}

This section incorporates with model of the gauge data analysis and water stature focus is used for producing the initial flooding probability index by creating the underlying rising water area. The 
extreme readings of water stature at a stream check speak to this present point's RT water stature amid the flood occasion. Information from each of the five checks was utilized in the model to redress for the vulnerability from neighborhood topological unevenness. With the readings at a given river point, the water height of each gauge is examined. The different between the reference water stature and greatest water tallness (recorded in Table 1) is one of the three classifications, which includes water body, overflowed, and non-flooded area.

The flooding probability was found by peak flow survey data over a different number of years, which is done with the help of MCMC technique. The existing method analyzed the inundation probability for finding the flood rate, which does not predict the earlier flood identity. In the proposed reconstructed model, the earlier prediction is done with the help of MCMC technique by posterior distribution function, which analyzed the inundation probability through peak flow of average flood rate from survey data. This result is viewed through the twitter API for identifying of flood possibilities. Return level of water flow is shown in Figure 2.

Here the three sections of proposed reconstruction model provide the result for flood identification, which creates awareness through social media. The MCMC performed with the average survey report for finding the flood possibility for the coming years. This system enables the earlier identification of regional area and city people by using tweet development API.

\subsection{Global Enhancement Section}

The global enhancement section was processed with land surface wetness and topographic attributes by coordinating the normalized water index surface, which is performed with the Enhanced Water Height Module. The IFPs $(a, b)$ represented the density at location $(a, b)$. The kernel function $K$ is used in the quadratic kernel, which is calculated as,

$K(d)=\frac{15}{16}\left(1-d^{2}\right)^{2}$

Table 1. Statistic analysis of five gauges from USGS dataset

\begin{tabular}{|c|c|c|c|c|c|}
\hline $\begin{array}{c}\text { United States } \\
\text { (Washington Rivers) }\end{array}$ & Gauge No. & $\begin{array}{c}\text { Flood } \\
\text { Stage }\end{array}$ & $\begin{array}{c}\mathbf{2 9 . 0 3 . 2 0 1 9} \\
\text { at time 't' }\end{array}$ & Percentage of mean & Percentage of median \\
\hline Walla river near Touchet & 14018500 & $13 \mathrm{ft}$ & $19: 00$ & $342 \%$ & $661 \%$ \\
\hline Fork Palouse river at Pullman & 13348000 & $7 \mathrm{ft}$ & $19: 15$ & $518 \%$ & $2134 \%$ \\
\hline Hangman Creek at Spokane & 12424000 & $11 \mathrm{ft}$ & $19: 45$ & $546 \%$ & $2932 \%$ \\
\hline Grande Ronde River at Troy & 13333000 & $10 \mathrm{ft}$ & $19: 00$ & $306 \%$ & $589 \%$ \\
\hline Snake River near Anatone & 13334300 & $20 \mathrm{ft}$ & $19: 15$ & $150 \%$ & $208 \%$ \\
\hline
\end{tabular}

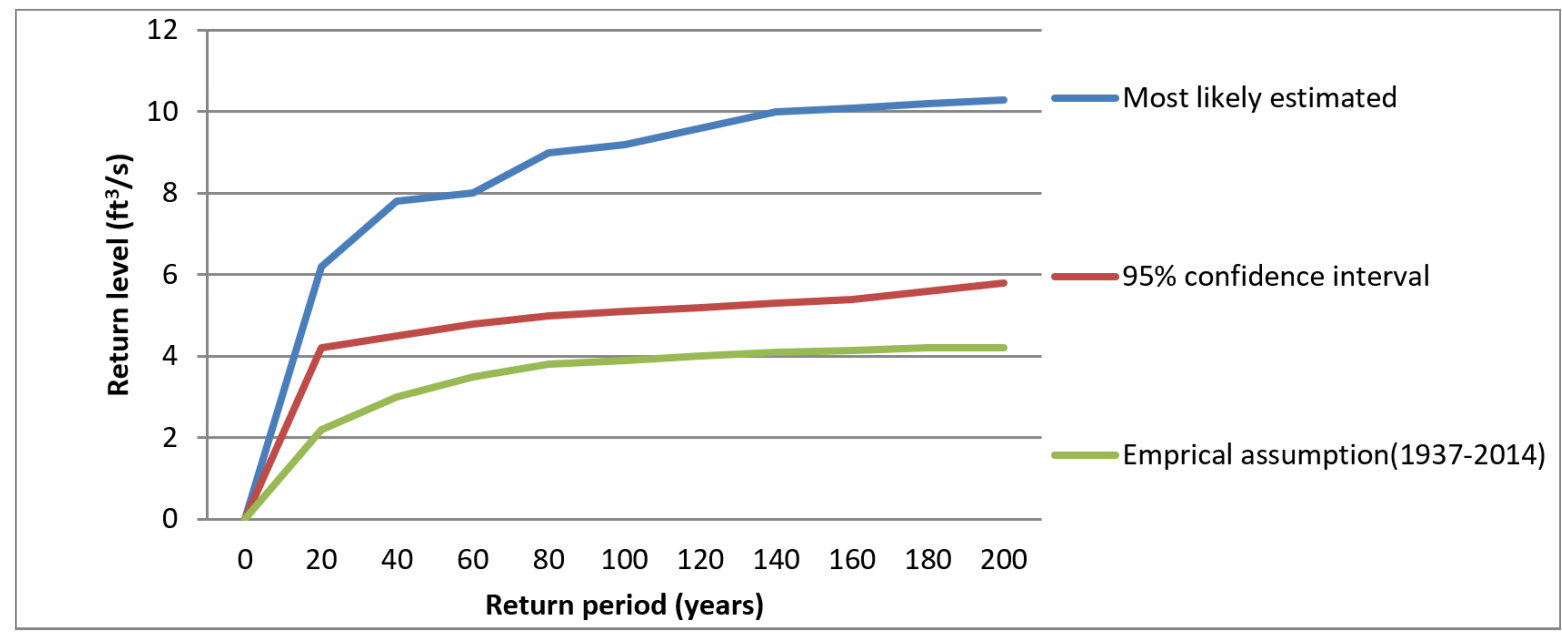

Figure 2. Water return level period (years vs feet) 
Where variable ' $d$ ' needs to meet a cutoff necessity $|\mathrm{d}| \leq 1$. The API-inferred water index is smoothened for utilizing a similar portion capacity and parameter settings. The $\mathrm{W}_{\text {index }}$ and $\mathrm{IFP}_{\mathrm{s}}$ are standardized by utilizing a maximumleast standardization of [ $\left[\begin{array}{ll}0 & 1\end{array}\right]$.

The IFS is disseminated reliably and recognizably for the entire extent of this estimate. Nevertheless, the $\mathrm{W}_{\text {index }}$ estimates are for the most part clustered for the entire range. To spread out these overflow estimates, an altered strategic stretch capacity is connected to $\mathrm{W}_{\text {index }}$.

$S_{W_{\text {index }}}=\frac{L}{1+e\left(k\left(W_{\text {index }}+a\right)\right)}$

Where $\mathrm{S}_{-} \mathrm{W}_{\text {index }}$ represents the extended water index, $\mathrm{k}$ is the coefficient of L-bend and it helps to estimate the midpoint. At last, an Enhanced Flood Probability is delivered by amassing the IFS $\mathrm{n}_{\mathrm{n}}$ and S_W $\mathrm{W}_{\text {index }}$ as follows:

$P_{\text {flood }}=I F S n+S_{-} W_{\text {index }}$

Where $\mathrm{P}_{\text {flood }}$ is Flooding Probability index, The IFP is Initial Flooding Probability and the $\mathrm{RF}_{\text {prob }}$ is Reconstructed Flooding Probability. With the use of Markov Chain Monte Carlo (MCMC) method, the frequency analysis is possible in the water height module. The MCMC technique is applied for future analysis not for present and past data, which performs the posterior functional distributions.

Tweet data comes from the direct accessing of the flood event with USGS map, which shows the flood point to determine the water level. Here the analysis result shows the five gauge values to determine the water height module and global enhancement module. The approach obtains the three separate sectional results and it utilizes the survey analysis for earlier prediction. After prediction of flood event, the water height module with image processing technique determines the water level; based on this, the tweet API shows the flooded area with secure accessing.

The flooding probability is used to determine the pointed tweets using posterior distribution function. This results in a better probability distribution function. These pointed tweets are obtained from the above-mentioned global flood predictor API (see Figure 3). During the flood event, the tweets are supplemented by twitter stream and the reconstruction of flooding probability is also improved. The secret key is verified on the API development website and MATLAB tweet performance.

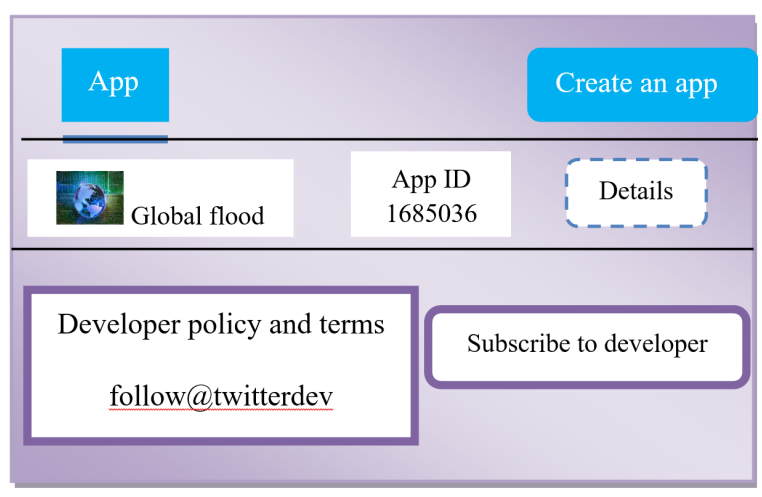

Figure 3. Global flood predictor API creation on tweets

\section{MCMC Technique}

Improved Markov Chain Monte Carlo technique is applied to perform posterior distribution function by fixing the average value computation references, which ease the system speed processing. Here it analyzes the future predicted value and it does not consider the past analysis. The MCMC technique is proposed with the threshold value to estimate the survey of flood events for past years. This information helps to identify the future prediction of flood event. The water peak of average value is discarded for ' $\mathrm{N}$ '. The initial peak value is calculated as,

$T_{\text {peak }}=\frac{N-1}{K}$

$T_{\text {peak }}=\frac{N+1-2 p}{K-p}$

Here, $p$ is the parameter depending on the peak value of discharge flood frequency. The calamity area based data execution of flood mapping is done with investigating the novel methodology with close continuous data by utilizing Twitter information in geospatial forms. The probability is calculated by,

$$
F_{\text {prob_freq }}=1-(1-m)^{N}
$$


The flood frequency information for each stream is analyzed for finding the posterior distribution and it is used for earlier flood prediction. In USGS database, the stream gauge map of flood event will calculate the flood frequency, which helps to analyze the function of MCMC easily. Each gauge reading is taken at 15-30 minute intervals. The existing method uses Gibbs sampling approach for flood probability prediction using flooded stream data. This takes every cycle's initial value to process since it takes a high computation time. The proposed method uses novel MCMC for reducing computation time as compared to the existing method.

\section{Results and Discussions}

Thus, the three modules of the proposed flood reconstruction model are designed to provide better result than the recent related works. The flooding probability points (tweets) are taken with the search area by means of a USGS geographical survey. Here the MCMC technique is improved by fixing the average value of water level on the survey report and it reduces the computation time. By the use of social media, the flood eventawareness is created among people by tweets of Twitter stream. It records every 15-minute interval and it reaches the people quickly. Here the Reconstructed Flood Probability index was evaluated by locally enhancing the EFP with the verified tweet points via a morphological operation. Inundation Flood Probability is merely based on water height readings at five gauges and DEM. The standard procedure makes these layers numerically identical. Input image is given in Figure 4. That is converted into grayscale image, which is shown in Figure 5.

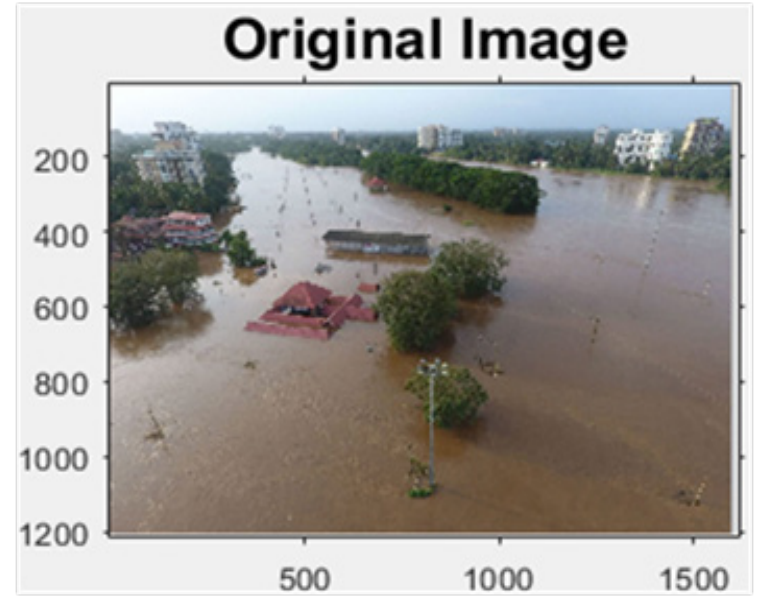

Figure 4. Input flooded image

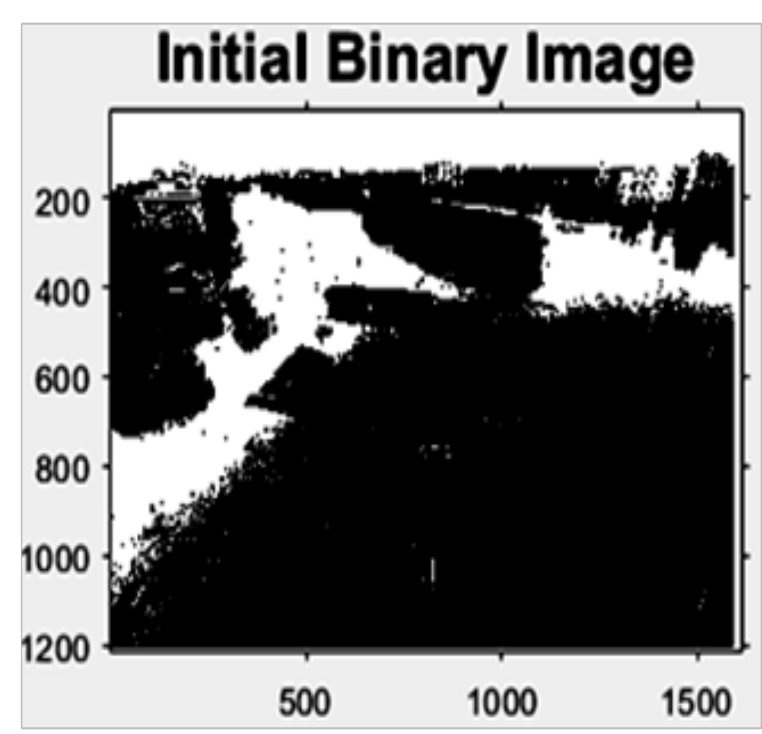

Figure 5. Grayscale image

A result provides the USGS (U.S. Geological Survey) flood map analysis, survey report analysis and tweet implementations. The proposed system is enhanced with the Markov Chain Monte Carlo technique for prior and posterior estimations.

Tweet API is developed to generate the key for accessing the information about flood event. Here the flood analysis report is shown in the tweets. By accessing the key twitter API shows the web page of flood event for knowing the water area; in this, the water index is determined by image processing approach to get the water level and the survey analysis shows the flood prediction state over a period of 100 years of flood events from USGS data. The boundary estimation using feret diameter calculation is given in Figure 6.

\section{Filled, Cleaned Binary Image with Boundaries and Feret Diameters}

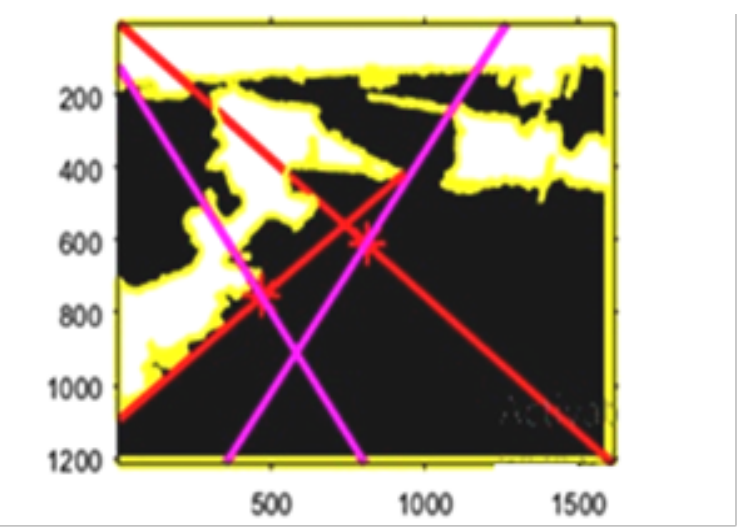

Figure 6. Feret diameter on the boundaries 
Proposed EFP by globally enhancing the satellite-extracted Normalized Water Index surface with the IFP via kernel smoothing, standardization and aggregation is done with the help of morphological process. Here the tweet points are analyzed to find the flood area based on performance of water index calculation. The performance analysis of flood inundation is given in the Table 2.

Table 2. Simulated result of Flood Inundation area

\begin{tabular}{|c|c|c|}
\hline S. No. & PARAMETERS & VALUES \\
\hline 1. & Long Slope & 0.7544 \\
\hline 2. & Perpendicular Slope & -1.3254 \\
\hline 3. & Max distance for blob & 2020.61 \\
\hline 4. & Perpendicular distance at midpoint & 1520.00 \\
\hline 5. & Average perpendicular width & 182.71 \\
\hline 6. & Area & 36918 \\
\hline 7. & Skew & -0.5147 \\
\hline
\end{tabular}

Table 3. Result comparison

\begin{tabular}{|c|c|c|}
\hline Parameter & $\begin{array}{c}\text { Existing flood } \\
\text { reconstruction model }\end{array}$ & Proposed work \\
\hline $\begin{array}{c}\text { Computation } \\
\text { time }\end{array}$ & $2.223 \mathrm{sec}$ & $1.222 \mathrm{msec}$ \\
\hline
\end{tabular}

The comparison results show (see Table 3) the overall computation time of three sectional modules and the proposed work utilizes the novel tweet implementation by accessing direct web page of flood event. The tweet implementation depends on the flood event of USGS data and it gives the direct map of gauge values. The results depend on the flood-based survey analysis and it obtained the real-time tweet implementation from the USGS dataset. The computation time is compared for how the three sectional modules are performing in a time-efficient manner. The consideration of

\section{REFERENCES}

Anon (2018). USGS Dataset Water science school. Available at: $<$ https://www.usgs.gov/special-topic/waterscience-school/science/floods-and-recurrence-intervals $>$.

Anterrieu, E. (2007). On the reduction of the reconstruction bias in synthetic aperture imaging radiometry, IEEE Transactions on Geoscience and Remote Sensing, 45(3), 592-601. various measurement vulnerabilities from nearby topological unevenness and defects of measure information itself effectively distinguishes the low-elevation overwhelmed territories. Thus the proposed model of flood-reconstruction model is designed to improve the earlier flood indication and creates awareness through social media. The simulated result represents the computation time of overall system.

\section{Conclusion and Future Work}

Thus, the proposed research work is concluded with realist images based on their results for flood prediction with social media. The existing analysis, takes the maximum duration and has delays in reaching people while the proposed research work is based upon updated Google based data fusion (USGS). This approach obtained efficient results by the use of Markov Chain Monte Carlo process with water report analysis based on USA database. This process avoids the delay of flood instruction to guide people so that they move to another place by raising social response awareness; which is one of the major advantages of the proposed research work. The local improvement with Real-Time tweets demonstrates that even a little amount of publicly supporting information can to a great extent improve the distinguishing proof of high flood likelihood zones (tweet points) in the context of a flood occasion. The proposed methodology could enable a wide range of future studies and it gives the best flood situation awareness in a city by social media (Twitter). In the future, the frequency analysis will be accurate by using novel algorithms. The immediate flood event results are being sent to the people by the use of various securities based social Media. The machine learning technique is applied for classification of flood prediction model.

Bazi Y., Bruzzone, L. \& Melgani. F. (2005). An unsupervised approach based on the generalized Gaussian model to automatic change detection in multi temporal SAR images, IEEE Transactions on Geoscience and Remote Sensing, 43(4), 874-887.

D’Amico., M., Manzoni A. \& Solazzi, G. L. (2016). Use of operational microwave link measurements 
for the tomographic reconstruction of 2-D maps of accumulated rainfall, IEEE Geoscience and Remote Sensing Letters, 13(12), 1827-1831.

Evans, T. L., Costa, M., Telmer, K. \& Silva, T. S. F. (2010) Using ALOS/PALSAR and RADARSAT-2 to map land cover and seasonal inundation in the Brazilian Pantanal, IEEE Journal of Selected Topics in Applied Earth Observations and Remote Sensing, 3(4), 560-575

Gaeta, R. \& Sereno, M. (2011). Generalized probabilistic flooding in unstructured peer-topeer networks, IEEE Transactions on Parallel and Distributed Systems, 22(12), 2055-2062.

Gailler A., Calais, E., Hebert, H., Roy, C. \& Okal, E. (2015). Tsunami scenarios and hazard assessment along the northern coast of Haiti, Geophysical Journal International, 203(3), 2287-2302.

Giustarini, L., Hostache, R., Kavetski, D., Chini, M., Corato, G., Schlaffer, S. \& Matgen, P. (2016). Probabilistic flood mapping using synthetic aperture radar data, IEEE Transactions on. Geoscience and Remote Sensing, 54(12), 6958-6969.

Huang, X., Li, X., Wang, C. \& Ning, H. (2019) Identifying disaster related social media for rapid response: a visual-textual fused $\mathrm{CNN}$ architecture, International Journal of Digital Earth. DOI: 10.1080/17538947.2019.1633425

Huang, X., Wang, C. \& Li, Z. (2018). A near real-time flood-mapping approach by integrating social media and post-event satellite imagery, Annals of GIS. DOI: 10.1080/19475683.2018.1450787

Huang, X., Wang, C., Li, Z., Ning, H. (2018). A Visualtextual fused approach to automated tagging of floodrelated tweets during a flood event, International Journal of Digital Earth, 12(11), 1248-1264.

Hung, X., Wang, C. \& Li, Z., (2018). Reconstructing flood inundation probability by enhancing near realtime imagery with real-time gauges and tweets," IEEE Transactions on Geoscience and Remote Sensing, 56(8), 4691-4701.

Khan, T. A., Alam, M. M., Shahid, Z. \& Su'ud, M. M. (2020) Investigation of flash flood on early basis: A Factual Comprehensive review, IEEE Access, 8, 19364-19380.

Landuyt, L., Van Wesemael, A., Schumann, G. J. P., Hostache, R., Verhoest, N. E. \& Van Coillie, F. M. (2018). Flood Mapping based on synthetic aperture radar: an assessment of established approaches, IEEE Transactions on Geoscience and Remote Sensing, 99, $1-18$.

Li., Z., Wang, C., Emrich, C. T. \& Guo, D. (2017). A novel approach to leveraging social media for rapid flood mapping: A case study of the 2015 South Carolina floods, Cartography and Geographic Information Science, 45(2), 97-110.

Nadeem, A., Abdul, R., Mujtaba, H., Saad, R., Malik, S. M., Mehwish, N., Alina, H., Nadeem, S. \& Maruf, P. (2019) Hierarchical colored petri-Net based MultiAgent System for flood monitoring, prediction and rescue (FMPR), IEEE Access, 7, 180544-180557.

Qavi, A., Khalil Ur, R., Zubair Arif, H. M., Farid, G., Khan, M. M. \& Waseem Bukhari, S. M. (2017) An approach to perform internal flooding PSA for power plants. In Proceedings of the International Conference on Innovations in Electrical Engineering and Computational Technologies, Karachi, Pakistan.

Saeed, T., Mylonas, Y., Pitsillides, A., Papadopoulou, V. \& Lestas, M. (2018). Modeling probabilistic flooding in VANETs for optimal rebroadcast probabilities, IEEE Transactions on Intelligent Transportation Systems, 99, 1-15.

Sarker C., Mejias Alvarez, L. \&Woodley, A. (2016) Integrating Recursive Bayesian Estimation with Support Vector Machine to Map Probability of Flooding from Multispectral Landsat Data. In Proceedings of the International Conference on Digital Image Computing: Techniques and Application, Gold Coast, Australia.

Shah-Mansouri, H., Pakravan, M. R. \& Khalaj, B. H. (2011). Analytical modeling and performance analysis of flooding in CSMA-based wireless networks, IEEE Transactions on Vehicular Technology, 60(2), 664-679.

Shen, X., Chen, Y., Zhang, Y., Zhang, J., Ge, Q., Dai, G. \& He, T. (2015). OppCode: Correlated opportunistic coding for energy-efficient flooding in wireless sensor networks, IEEE Transactions on Industrial Informatics, 11(6), 1631-1642.

Takala, M., Ikonen, J., Luojus, K., Lemmetyinen, J., Metsämäki, S., Cohen, J. \& Pulliainen, J. (2017). New snow water equivalent processing system with improved resolution over europe and its applications in hydrology, IEEE Journal of Selected Topics in Applied Earth Observations and Remote Sensing, 10(2), 428-436. 
Wang C., Li, Z. \& Huang, X. (2018). Geospatial Assessment of Wetness Dynamics in the October 2015 SC Flood with Remote sensing and Social Media, Journal of Southeastern Geographer, 58(2), 164-180.

Wang, S., Huang, G. \& Baetz, B. W. (2015). An inexact probabilistic-possibilistic optimization framework for flood management in a hybrid uncertain environment, IEEE Transactions on Fuzzy Systems, 23(4), 897-908.

Xu, X., Wang, J. \& Yu, Y. (2020). Alarm Evant Prediction from Historical alarm flood sequences based on Bayesian estimators, IEEE Transactions on Automation, 17(2), 1070-1075.
Youngjoo, K., Jonggeol, P. \& Kazuhiko, F. (2014). Near Real-Time Flood volume estimation from MODIS Time-series imagery in the Indus River Basin, IEEE Journal of Selected topics in Applied Earth Observations and Remote Sensing, 7(2), 578-586.

Zhu, Z., Yong, B., Ke, L., Wang, G., Ren, L. \& Chen, $X$. (2018). Tracing the error sources of global satellite mapping of precipitation for GPM (GPM-GSMaP) over the Tibetan Plateau, China, IEEE Journal of Selected Topics in Applied Earth Observations and Remote Sensing, 11(7), 2181-2191. 